\title{
CURIE TEMPERATURE OF DISORDERED ALLOYS
}

\section{A.K. Arzinikov and L.V. Dobysheva}

Phys. \& Techn. Inst., Kirov str, 132, Izhevsk, 426001, Russia

The concentrational behavior of the Curie temperature that describes the experiments for disordered alloys $\mathrm{Fe}-\mathrm{M}(\mathrm{M}=\mathrm{Al}, \mathrm{Si}, \mathrm{Ga}, \mathrm{Sn})$ at concentrations of $M$ lower than 10 at.\% was obtained in the framework of the Hubbard model. The influence of the spin and charge fluctuations was investigated.

PACS numbers: 71.10.Fd, 75.10.Lp, 75.50.Bb

The analysis of numerous crystallographic, Mössbauer, and magnetic experiments on the disordered alloys $\mathrm{Fe}-\mathrm{M}(\mathrm{M}=\mathrm{Al}, \mathrm{Si}, \mathrm{Ga}, \mathrm{Sn})$ allowed us to describe their general qualitative peculiarities in the framework of the two-band Hubbard model $[1,2]$. Subsequent reduction of the Hamiltonian is justified at concentrations of $\mathrm{M}$ lower than 10 at.\% [3], the concentrational variations of the hybridization of two bands being neglected. Really, the fact that some features in the behavior of the Curie temperature $T_{\mathrm{C}}$ (Fig. 1) and of the magnetic moment that practically is constant on the Fe atom at low concentrations [4-6] are common to different alloys in spite of large differences in atomic radii and electron configuration of metalloid, is indicative of limited number of the factors which determine $T_{C}$ and the moment. This allows the Hamiltonian to be written in the following form:

$$
\begin{aligned}
& H=\sum_{i \sigma} \varepsilon_{i} c_{i \sigma}^{+} c_{i \sigma}+\sum_{i j} t_{i j} c_{i \sigma}^{+} c_{j \sigma}+U \sum_{i} n_{i \uparrow} n_{i \downarrow}=H_{0}+H_{\mathrm{int}}, \\
& H_{\mathrm{int}}=\sum_{i \sigma} \varepsilon_{i} c_{i \sigma}^{+} c_{i \sigma}+U \sum_{i} n_{i \uparrow} n_{i \downarrow},
\end{aligned}
$$

where $\varepsilon_{i}$ takes the value $\varepsilon^{\mathbf{A}}$ for the metalloid atoms and $\varepsilon^{\mathbf{B}}$ for the iron atoms, $\left|\varepsilon^{\mathrm{A}}-\varepsilon^{\mathrm{B}} / W\right| \gg 1$ being fulfilled, $W$ is the band width. $c_{i \sigma}^{+}\left(c_{i \sigma}\right)$ are the creation (destruction) operators of the electrons with spin $\sigma$ at site $i, n_{i \sigma}=c_{i \sigma}^{+} c_{i \sigma}, U$ is the constant of intraatomic interaction of electrons. Choosing $t_{i j}$ and $U$ the same for both $\mathrm{A}$ and $\mathrm{B}$ atoms (corresponding to $\mathrm{Fe}$ ) we do not mistake noticeably, because the $d$-band of the metalloid is far higher than the Fermi level and remains unfilled. The Hamiltonian (1) is a single-band one. As shown in Ref. [7], the calculations in a single-band model followed by the renormalization of the free energy and the magnetic moment take into account both the degeneracy over the orbital 
quantum number and the IIund rule. Using the equality $n_{i \uparrow} n_{i \downarrow}=n_{i}^{2} / 4-S_{i z}^{2}$, where $n_{i}=n_{i \uparrow}+n_{i \downarrow}, S_{i z}=\left(n_{i \uparrow}-n_{i \downarrow}\right) / 2$ we write the partition function of the system in the interaction representation, and pass to the continual integral by the Stratonovitch-IIubbard equality [8]

$$
\begin{aligned}
Z= & \int\left(\prod_{l} \delta x_{l}(\tau) \delta v_{l}(\tau)\right) \exp \left[-\frac{\pi}{\beta} \int_{0}^{\beta} \mathrm{d} \tau \sum_{l}\left[x_{l}^{2}(\tau)+v_{l}^{2}(\tau)\right]\right] Z\left\{x_{l}(\tau), v_{l}(\tau)\right\} \\
& Z\left\{x_{l}(\tau), v_{l}(\tau)\right\}=\operatorname{Tr}\left\{\operatorname { e x p } ( - \beta \tilde { H } _ { 0 } ) T _ { \tau } \operatorname { e x p } \left[-\frac{1}{\beta} \int_{0}^{\beta} \mathrm{d} \tau\right.\right. \\
& \left.\left.\times \sum_{l}\left[2 \sqrt{\pi \beta U} v_{l}(\tau) S_{z}(\tau)+\sqrt{\pi \beta U} \mathrm{i} x_{l}(\tau) n_{l}(\tau)+\beta \varepsilon_{l} n_{l}(\tau)\right]\right]\right\}
\end{aligned}
$$

This transition is possible since all the operators in $H_{\text {int }}$ commute, including the disorder term. Using the single-site and static approximations, taking into consideration the fact that charge fluctuations $x_{l}$ are quick variables as compared to the spin ones $v_{l}$, we perform the integration with respect to $x_{l}$. In this work the quantity $w_{l}$ associated with the charge fluctuations was chosen in two ways. In the first case we neglected the charge fluctuations, assuming only that the Fe site was electroneutral on the average. In the second case the saddle-point method was used for taking into account the charge fluctuations [8].

$Z=Z_{0} \int\left(\prod_{l} \mathrm{~d} v_{l}\right) \exp \left\{-\pi \sum_{l}\left(v_{l}^{2}-w_{l}^{2}\right)-\frac{\beta}{\pi} \int_{-\infty}^{+\infty} \mathrm{d} z \frac{\operatorname{Tr} \operatorname{Im} \ln \left(1-G^{0} V\right)}{\exp [(z-\mu) / T]+1}\right\}$,

here $V$ is the matrix over sites $l, l^{\prime}$, consisting of $2 \times 2$ blocks

$$
V_{l^{\prime}}=\left(\begin{array}{cc}
\sqrt{\frac{\pi U}{\beta}}\left(w_{l}+v_{l}\right)+\varepsilon_{l} & 0 \\
0 & \sqrt{\frac{\pi U}{\beta}}\left(w_{l}-v_{l}\right)+\varepsilon_{l}
\end{array}\right) \Delta_{l l^{\prime}}=\tilde{V}_{l},
$$

$\Delta_{l \prime}$ is the Kronecker delta, $G_{l l^{\prime}}^{0}$ is the matrix of the Green function of the system with the IIamiltonian $H_{0}$. Apart from $\varepsilon_{l}$, the formula (4) is a standard one among the representations of the Ilubbard model by the continual integral.

The coherent potential approximation gives the equation for the self-energies $\Sigma^{\dagger}(z)$ and $\Sigma^{\downarrow}(z)$

$$
\int_{-\infty}^{+\infty} \mathrm{d} v \int_{-\infty}^{+\infty} \mathrm{d} \varepsilon_{l} t_{l} W\left(v, \varepsilon_{l}\right)=0
$$

where $t_{l}=\left(\tilde{V}_{l}-\Sigma\right)\left[1-g\left(\tilde{V}_{l}-\Sigma\right)\right]^{-1} . W\left(v, \varepsilon_{l}\right)$ is the probability density of the distribution of the spin fluctuations $v$ and the parameter $\varepsilon_{l}$ at site $l$, that was found from the partition function. The substitution of variables $v=v_{l} \sqrt{\pi U T}$ and $w=w_{1} \sqrt{\pi U T}$ gives rise to

$$
\begin{aligned}
& W\left(v, \varepsilon_{l}\right)=C\left[x \delta\left(\varepsilon^{\mathrm{A}}-\varepsilon_{l}\right)+(1-x) \delta\left(\varepsilon^{\mathrm{B}}-\varepsilon_{l}\right)\right] \\
& \quad \times \operatorname{cxp}\left\{-\frac{1}{U T}\left(v^{2}-w^{2}+\frac{U}{\pi} \int_{-\infty}^{\mu} \mathrm{d} z \operatorname{Tr}_{\sigma} \operatorname{Im} \ln \left[1-g\left(\tilde{V}_{l}-\Sigma\right)\right]\right)\right\},
\end{aligned}
$$


where $C$ is the normalization factor. The equality (5) together with the condition for the chemical potential constitutes a closed system of equations.

The used model density of states, simulating this of $\mathrm{Fe}$, is shown in the inset of Fig. 1. The parameter $U$ was chosen so that the magnetic moment of $\mathrm{Fe}$ at $T=0$ and $x=0$ was close to the experimental value, the number of $d$-electrons below the Fermi level with allowance made for the degeneracy being equalled to 7.425 and 7.25. The density of states and all the parameters chosen were close to those used in other papers $[8,9]$. At the approximations used here a complete quantitative agreement of the experimental and calculated values cannot be expected, so the calculated values reduced to the Curie temperature $T_{\mathrm{C}}^{0}$ at $x=0$ are reported. The Curie temperature was detcrmined as the temperature of the vanishing of the magnetic moment.

The concentrational dependences obtained are shown in Fig. 1 (curves 1-3). There also are $T_{\mathrm{C}}(x) / T_{\mathrm{C}}^{0}$ for the IIeisenberg model with non-magnetic impurities (curve 4 ) and the extrapolation $T_{\mathrm{C}}(x) \approx J_{\text {eff }}(x)$, where $J_{\text {eff }}(x)$ is the effective exchange interaction obtained at $T=0$ (curve 5). The dependences 1-3 are evidently in good agreement with the experimental data, unlike the curves 4-5. This indicates a basic unsuitability of the IIeisenberg models with non-magnetic impurities and of the interpolation $T_{\mathrm{C}}(x) \approx J_{\mathrm{eff}}(x)$ for describing $T_{\mathrm{C}}(x)$ of the above alloys.

A more detailed description of $T_{\mathrm{C}}(x)$ depending on the metalloid type requires considering the second band. The possibility of such a description is due to the fact that the $T_{\mathrm{C}}(x)$ slope depends on the number of $d$-electrons (curves 1,3 ) which is determined by the $s d$-hybridization value [1] and increases as the impurity concentration rises. It should be noted that taking into account the charge fluctu-

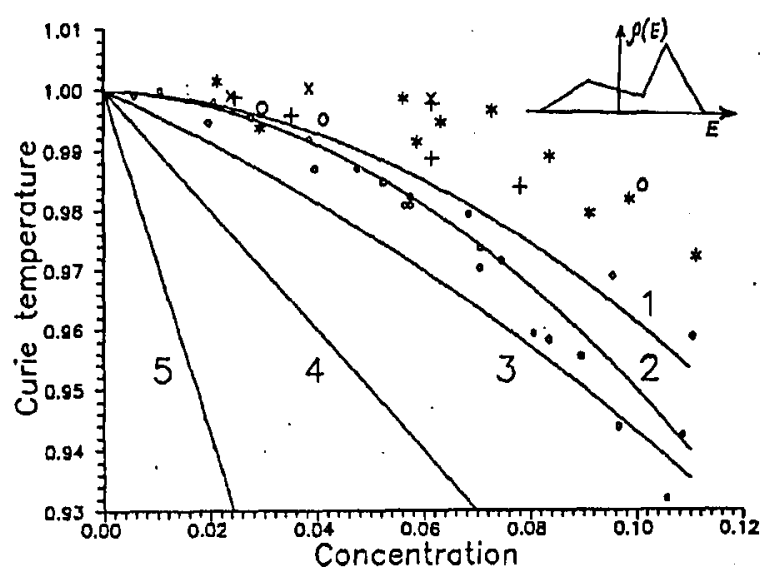

Fig. 1. The concentrational dependence of the Curie temperature of disordered alloys: ( $\star$ Fe-Al [10-12, 6] (o) Fe-Si [10-11, 13-15, 5], (O) Fe-Ga [12], ( $\times$ ) Fe-Sn [10], (+) Fe-Sn [3]; curve $1-$ number of $d$-electrons, $n_{d}=7.425$, without taking into account the charge fluctuations; $2-n_{d}=7.425$, with taking into account the charge fluctuations; $3-n_{d}=7.25$, without taking into account the charge fluctuations; $4-$ the Heisenberg model, 5 - the model $T_{\mathrm{C}}(x) \approx J_{\text {eff }}(x)$. 
ations also leads to a shift of $T_{\mathrm{C}}(x)$ (curve 1,2 ), so the choice of approximations for the detailed description requires a more careful analysis of the experimental data on the charge states of the system.

\section{References}

[1] A.K. Arzhnikov, L.V. Dobysheva, J. Magn. Magn. Mater. 117, 87 (1992).

[2] A.K. Arzhnikov, L.V. Dobysheva, Phys. Lett. A 195, 176 (1994).

[3] A.K. Arzhnikov, L.V. Dobysheva, E.P. Yelsukov, A.V. Zagaynov, JETF 110, 1127 (1996).

[4] E.P. Elsukov, V.A. Barinov, V.P. Galahov, E.E. Yurchekov, A.E. Ermakov, Fiz. Met. Metalloved. 55, 337 (1983).

[5] E.P. Elsukov, G.N. Konygin, V.A. Barinov, E.V. Voronina, J. Phys., Condens. Matter 4, 7597 (1992).

[6] E.P. Yelsukov, E.V. Voronina, V.A. Barinov, J. Magn. Magn. Mater. 115, 271 (1992).

[7] Y. Kakehashi, Phys. Rev. B 34, 3243 (1986).

[8] T. Moriya, Spin Fluctuations in Itinerant Electron Magnetism, Springer-Verlag, Heidelberg 1985.

[9] V.I. Grebennikov, Yu.I. Prokopyev, O.B. Sokolov, E.A. Turov, Fiz. Met. Metalloved. 52, 679 (1981) (in Russian).

[10] M. Fallot, Ann. Phys. 6, 305 (1936).

[11] D. Parsons, W. Sucksmith, J.k. Thompson, Philos. Mag. 3, 1174 (1958).

[12] I. Vincze, L. Cser, Phys. Stalus Solidi B 50, 709 (1972).

[13] S. Arais, Phys. Status Solidi 11, 121 (1965).

[11] W. Pepperhoff, H.-H. Ettwig, Z. Phys. 22, 497 (1967).

[15] D. Meinhardt, O. Krisement, Arch. Eisenhuttenw. 36, 293 (1965). 